\title{
A violência contra o idoso e a assistência da enfermagem na identificação e prevenção
}

\author{
Violence against the elderly and nursing care in the identification and prevention \\ Violencia contra el adulto mayor y cuidados de enfermería em la identificación y prevención
}

\section{Nathália de Deus Nunes e Nunes ${ }^{1 *}$, Sebastião Jorge da Cunha Gonçalves ${ }^{2}$, Alessandra da Silva Souza ${ }^{3}$, Jannaina Sther Leite Godinho Silva ${ }^{4}$, Adiel Queiroz Ricci ${ }^{5}$, Carolina de Lourdes Julião Vieira ${ }^{6}$.}

Como citar esse artigo. Nunes, NDD; Gonçalves SJC; Souza, AS; Silva, JSLG; Ricci,AQ; Vieira, CLJ.Aviolência contra o idoso e a assistência da enfermagem na identificação e prevenção. Revista Pró-UniverSUS. 2021 Jan./Jun.; 12 (1) SUPLEMENTO: 116-121.

\section{Resumo}

Com o aumento da expectativa de vida, um problema social complexo vem emergindo, a violência contra o idoso. Os profissionais de enfermagem estão em contato diário com a população idosa, sendo estes responsáveis na intervenção dos casos de violência. O objetivo deste estudo é analisar a atuação do enfermeiro (a) na identificação e na prevenção da violência contra pessoa idosa. E apresentar os programas de atenção à saúde e as políticas públicas que envolvem a proteção do idoso. Tratase de um estudo de revisão da literatura, no qual utilizou-se para o levantamento bibliográfico, a Biblioteca Virtual de Saúde (BVS) e as seguintes bases de dados: SCIELO, LILACS e BDENF, os descritores foram: maus-tratos ao idoso e enfermagem. Foram selecionados e utilizados no estudo 17 artigos. Através da análise dos dados, observou-se que os idosos violentados, em sua maioria, tem o seguinte perfil: gênero feminino, estado civil: casadas, sendo algum membro familiar o principal agressor. Através da assistência da enfermagem, pode-se: identificar as agressões, realizar o acolhimento à vítima e notificar. No entanto o enfermeiro (a) encontra dificuldades para realizar as notificações. Conclui-se que a enfermagem tem um papel fundamental na proteção ao idoso. Fazendo-se necessária a capacitação constante dos profissionais, para que tenham conhecimento de como proceder frente a esses casos. Muitos protocolos e leis não são seguidos, por isso há necessidade de um controle mais rigoroso das notificações e denúncias, além da necessidade de investimentos na criação de vigilâncias e programas de assistência ao idoso.

Palavras-chave: Enfermagem; Idoso; Violência

\begin{abstract}
With the increase in life expectancy, a complex social problem has emerged, violence against the elderly. Nursing professionals are in daily contact with the elderly population, who are responsible for the intervention of cases of violence. The aim of this study is to analyze the role of nurses in the identification and prevention of violence against the elderly. And to present health care programs and public policies that involve the protection of the elderly. This is a literature review study, in which the Virtual Health Library (VHL) and the following databases were used for the bibliographic survey: SCIELO, LILACS and BDENF, the descriptors were: mistreatment to the elderly and nursing. 17 articles were selected and used in the study. Through data analysis, it was observed that the majority of assaulted elderly people have the following profile: female gender, marital status: married, with some family member being the main aggressor. Through nursing care it is possible to: identify the aggressions, welcome the victim and notify. However, the nurse finds it difficult to carry out the notifications. It is concluded that nursing has a fundamental role in protecting the elderly. Making constant training of professionals necessary, so that they have knowledge of how to proceed in these cases. Many protocols and laws are not followed, and there is a need for stricter control of notifications and complaints, in addition to the need for investments in the creation of surveillance and assistance programs for the elderly. Keywords: Nursing; Seniors; Violence.
\end{abstract}

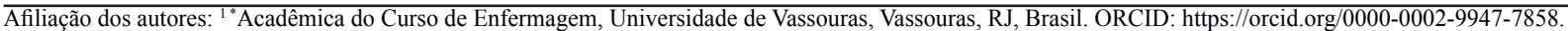

${ }_{2}^{2}$ Mestre em Psicologia pela Faculdade Jesuíta de Filosofia e Teologia. Docente do Curso de Enfermagem da Universidade de Vassouras, Vassouras, RJ, Brasil. E-mail: sjcunha@uol. com.br ORCID: https://orcid.org/0000-0002-4228-4641.

${ }^{3}$ Mestre em Enfermagem pela Universidade de Vassouras. Docente do Curso de Enfermagem da Universidade de Vassouras, Vassouras, RJ, Brasil. ORCID: https://orcid.org/00000002-9009-9774;

${ }^{4}$ Mestre em Enfermagem pela Universidade Federal Fluminense. Docente do Curso de Enfermagem da Universidade de Vassouras, Vassouras, RJ, Brasil. ORCID: https://orcid. org/0000-0002-8308-2093.

${ }^{5}$ Mestre em Estudo de Linguagem pela Universidade Federal Fluminense. Docente do Curso de Enfermagem da Universidade de Vassouras, Vassouras, RJ, Brasil. ORCID: https:// orcid.org/0000-0003-2761-2499.

${ }^{6}$ Doutora em Saúde pela Universidade Federal de Juiz de Fora. Docente do Curso de Enfermagem da Universidade de Vassouras, Vassouras, RJ, Brasil.ORCID: https://orcid. org/0000-0002-5785-5988.

* Email de correspondencia: nathydnnunes2017@gmail.com

Recebido em: 22/01/21. Aceito em: 18/05/21. 


\section{Resumen}

Con el aumento de la esperanza de vida, ha surgido un problema social complejo, la violencia contra personas importantes. Los profesionales de enfermería están en contacto diario con la población anciana, quienes son los encargados de la intervención de los casos de violencia. El propósito de este estudio es analizar el papel de la enfermera en la identificación y prevención de la violencia contra las personas mayores. Y presentar programas de salud y políticas públicas que involucren la protección de personas mayores. Se trata de un estudio de revisión de literatura, en el cual se utiliza la Biblioteca Virtual en Salud (BVS) y las siguientes bases de datos para el relevamiento bibliográfico: SCIELO, LILACS y BDENF, los descriptores fueron: la violencia al mayores y enfermera. seleccionó y utilizó 17 artículos en el estudio. Mediante el análisis de los datos se observó que la mayoría de las personas más asaltado tenían el siguiente perfil: sexo femenino, estado civil: casado, algún familiar, principal agresor. A través del cuidado de enfermería es posible: identificar agresiones, apoyo a la víctima y notificar. Sin embargo, la enfermera tiene dificultades para realizar notificaciones. Se concluye que la enfermera stiene un papel fundamental en la protección de las personas mayores. Como existe la necesidad de una formación constante de los profesionales, para que yo tenga más conocimiento de cómo proceder en estos casos. Muchos protocolos y leyes no se cumplen, ya que existe la necesidad de un control más estricto de las notificaciones y denuncias, además de la necesidad de inversiones en la creación de programas de vigilancia y atención a las personas más grandes.

Palabras clave: Enfermería; Vejez; Violencia.

\section{Introdução}

A população idosa vem aumentando consideravelmente nos últimos anos, sendo este um fenômeno mundial. No Brasil as estatísticas não são diferentes, pesquisas apontam que o Brasil tem mais de 28 milhões de idosos, número que representa $13 \%$ da população do país, e este percentual tende a crescer nas próximas décadas. ${ }^{1}$ Os registros sobre a violência contra a pessoa idoso consequentemente também aumentaram, tornando-se um problema social grave, que envolve todas as classes sociais, independentes de classe econômica, etnia e religião. ${ }^{2}$

Com as mudanças provenientes da idade, o idoso começa a apresentar sinais fisiológicos do envelhecimento, alterações no estilo de vida e problemas de saúde, e consequentemente começam a precisar de auxílio de outros para exercer atividades comuns que antes eram realizadas por eles mesmos. ${ }^{3}$ A pessoa idosa passa a ser mais vulnerável à violência quando começa a depender de maiores cuidados físicos ou apresentam dependência física ou mental. Quanto mais dependente o idoso fica, mais a escala de vulnerabilidade aumenta. O meio familiar conturbador e cuidadores despreparados pioram está situação..$^{4-5}$

Entre os vários tipos de violência sofrida pelo idoso, as que se destacam são: abuso físico (no qual é usada a força física); emocional ou psicológica (são as agressões verbais ou por gestos); a negligência (é a falta de cuidado e atenção por parte do responsável com a vítima); autonegligência (é a negação de prover a si mesmo do cuidado adequado); o abandono (é a ausência da pessoa responsável pelo cuidado e custódia da vítima); o financeiro (é o uso indevido dos recursos financeiros do idoso); e o abuso sexual (é usar a vítima para obter excitação sexual). ${ }^{6-7}$

A notificação realizada pelos profissionais de saúde em relação a violação ao idoso auxilia na avaliação epidemiológica do problema e contribui no desenvolvimento de programas relacionados a proteção do idoso. Estas notificações são frequentemente difíceis de serem realizadas, pois na maioria das vezes o agressor é um familiar. Assim, em defesa do agressor (membro da família), o idoso acoberta e ainda justifica a agressão sofrida. Resultando em uma questão complexa e silenciosa, que dificulta a conduta apropriada de intervenção, sendo um fenômeno desafiador para as políticas sociais.

Diante deste cenário, se fez necessária a obtenção de direitos, leis e a criação de políticas públicas direcionadas aos idosos. Esses direitos são estabelecidos pela Constituição Federal de 1988, pela Política Nacional do Idoso de 1994, pelo Estatuto do Idoso de 2003 e no departamento de saúde está garantido pela Política Nacional do Idoso de 1999, a qual inclui uma atualização no ano $2006 .{ }^{8}$ Porém, mesmo diante das legislações que garantem a segurança e bem estar dos idosos, a violência contra eles ainda persiste, sendo assim um problema de saúde pública, pois causa sérios agravos à saúde do indivíduo, da família e da sociedade, elevando os custos emocional e social. ${ }^{9}$

A violência contra a pessoa idosa é um problema complexo e de difícil resolução, podendo passar despercebido na maior parte dos casos, com isso o profissional de enfermagem pode encontrar dificuldades em identificar os sinais de violência e maus-tratos ao idoso assistido por ele. E os trabalhos relacionados ao tema, tornam-se uma contribuição para que os enfermeiros possam adquirir conhecimento e ter um olhar mais específico e voltado na identificação e prevenção da violência. ${ }^{2}$

Este estudo tem como objetivo analisar a assistência do enfermeiro (a)na identificação e prevenção da violência sofrida pela pessoa idosa. E apresentar os programas de atenção à saúde e as políticas públicas que envolvem a proteção do idoso 


\section{Materiais e Métodos}

Trata-se de um estudo de revisão da literatura. Quanto a este método, a revisão da literatura permite identificar, conhecer e acompanhar o desenvolvimento de determinado campo de conhecimento, levando perspectivas e sugestões para futuros trabalhos. ${ }^{10}$

Utilizou-se no levantamento bibliográfico, a Biblioteca Virtual de Saúde (BVS) e as seguintes bases de dados: SCIELO, LILACS e BDENF, os descritores foram: Maus-Tratos ao Idoso e Enfermagem. Entre os 34 artigos encontrados, 17 foram selecionados por estarem dentro dos critérios e foram utilizados no estudo. Foi feito uma leitura prévia do título e resumo de todos os artigos encontrados para selecionar somente os que se enquadram nos critérios de artigos publicados nos últimos 10 anos, disponíveis na íntegra e em língua portuguesa e relacionados a temática violência ao idoso e enfermagem.

\section{Resultados e Discussão}

No processo da análise dos artigos selecionados para o estudo. Após obedecer os critérios de exclusão e inclusão, foram selecionados um total de 17 artigos publicados em periódicos nacionais nos quais abaixo foram citados (Tabela 1).

Observou-se que a Revista de Enfermagem UFPE (37\%), teve uma maior contribuição para a construção deste artigo. Seguido da Revista Brasileira de Enfermagem (25\%) e a Escola Anna Nery Revista Brasileira de Enfermagem (25\%).

Constata-se que a temática violência contra os idosos, ainda é pouco discutida e pontuada. A violação contra o idoso tornou-se um problema de saúde pública e as publicações científicas no Brasil sobre o tema são escassas. Saber identificar as vítimas, em que meio estão e quais as suas particularidades, tanto no que se refere ao tipo de violência, como na identificação dos agressores, são informações essenciais para a ação do Estado, a criação de políticas públicas e até mesmo para garantir um atendimento de saúde adequado por parte dos profissionais dessa área. ${ }^{11}$

Enfatiza-se a necessidade de ações intersetoriais nos coletivos urbanos e a sensibilização dos profissionais de saúde, para que atendam as vítimas de forma clara e interdisciplinar com os demais setores sociais, e tenham métodos claros de participação para proteger os idosos e punir os responsáveis. ${ }^{11}$ Nesse sentido, a capacidade do sistema de saúde também ajuda a reverter o alto índice de mortalidade causado por essa condição e suas consequências: medo, alienação, estresse póstraumático e até mesmo a depressão. ${ }^{12}$

A violência deve ser tratada de forma responsável, conscientizando a sociedade a denunciar os casos, é preciso um aprendizado sobre a cultura de se penalizar os agressores. Dando a devida importância à dignidade humana e expandindo a qualidade de vida, da saúde comunitária e de uma sociedade livre de violências. ${ }^{13-14}$

Em relação aos tipos de estudo utilizados nos artigos, a tabela 2 a seguir descreve o método usado.

Tabela 1. Periódicos dos artigos públicados entre os anos de 2010 e 2020.

\begin{tabular}{ll}
\hline Periódico & N (\%) \\
\hline Revista Ciência Saúde Coletiva & $1(1,3 \%)$ \\
Revista Brasileira de Enfermagem & $2(25 \%)$ \\
Escola Anna Nery Revista Brasileira de Enfermagem & $2(25 \%)$ \\
Revista Pesquisa Cuidado Fundamental & $1(1,3 \%)$ \\
Revista Brasileira de Geriatria e Gerontologia & $1(1,3 \%)$ \\
Revista Baiana de Enfermagem & $1(1,3 \%)$ \\
Revista de APS & $1(1,3 \%)$ \\
Revista Gaúcha de Enfermagem & $1(1,3 \%)$ \\
Revista Kairós & $1(1,3 \%)$ \\
Revista Estudos interdisciplinares sobre o envelhecimento & $1(1,3 \%)$ \\
Revista Enfermagem UERJ & $1(1,3 \%)$ \\
Revista Texto \& Contexto Enfermagem & $1(1,3 \%)$ \\
Revista de Enfermagem UFPE & $3(37 \%)$ \\
\hline
\end{tabular}


Tabela 2. Os tipos de estudo dos dos artigos públicados entre os anos de 2010 e 2020.

\begin{tabular}{ll}
\hline Tipo de Estudo & N (\%) \\
\hline Estudo descritivo & $5(30 \%)$ \\
Estudo exploratório- descritivo & $3(20 \%)$ \\
Revisão integrativa & $1(5 \%)$ \\
Estudo epidemiológico & $1(5 \%)$ \\
Estudo reflexivo & $1(5 \%)$ \\
Pesquisa qualitativa & $3(20 \%)$ \\
Pesquisa quantitativa & $2(10 \%)$ \\
Não informado & $1(5 \%)$ \\
\hline
\end{tabular}

Fonte: Autor, 2021

Os resultados obtidos na análise destes estudos apontam que 5 dos artigos selecionados são descritivos (30\%), tendo o intuito de analisar e descrever os tipos de maus-tratos contra os idosos e analisar as práticas de enfrentamento realizadas pelo profissional de enfermagem. Já 3 artigos são estudos de caráter exploratório- descritivo (20\%) que através de estudo de casos, buscou-se o perfil da vítima e do seu agressor relatando as causas e efeitos da violência, os outros 3 artigos foram de pesquisa qualitativa $(20 \%)$ no qual investigaram a atuação do enfermeiro e se este está preparado para realizar as notificações, 2 artigos de pesquisa quantitativa $(10 \%)$ no qual as informações sobre o tema foram classificadas e analisadas por meios estatísticos. Nos outros artigos, 1 artigo foi revisão integrativa $(5 \%)$, o estudo foi realizado através de um levantamento bibliográfico, 1 artigo sobre estudo epidemiológico $(5 \%)$ no qual através de dados foi feita a distribuição que analisou o determinante da violência e o perfil das vítimas, e 1 artigo reflexivo (5\%) no qual o profissional de enfermagem relatou sua experiência sobre as notificações de violência sofrida pelo idoso. E 1 artigo (5\%) não foi informado o seu método de estudo.

Percebe-se que os conteúdos das investigações se limitaram em conhecer o perfil das vítimas e a prevalência do tipo de abuso. No entanto, poucos estudos tentaram compreender as principais motivações dos agressores, estudos de intervenções com equipes de saúde com propostas de inovação e estratégias de prevenção e diagnóstico. São poucas as pesquisas específicas sobre outros tipos de violência, como discriminação envolvendo a terceira idade, violência institucional, social e econômica. Os idosos muitas vezes sofrem abusos financeiros, como fraudes, que são praticadas principalmente por bancos e instituições de saúde, bem como lojas em geral. Nenhuma pesquisa foi encontrada para estender esse fenômeno, ou mesmo a frequência com que ele ocorre, levando a um maior encobrimento do problema ${ }^{15}$

Através dos artigos, foi possível analisar os profissionais que mais publicaram estudos sobre o tema (Figura 1).

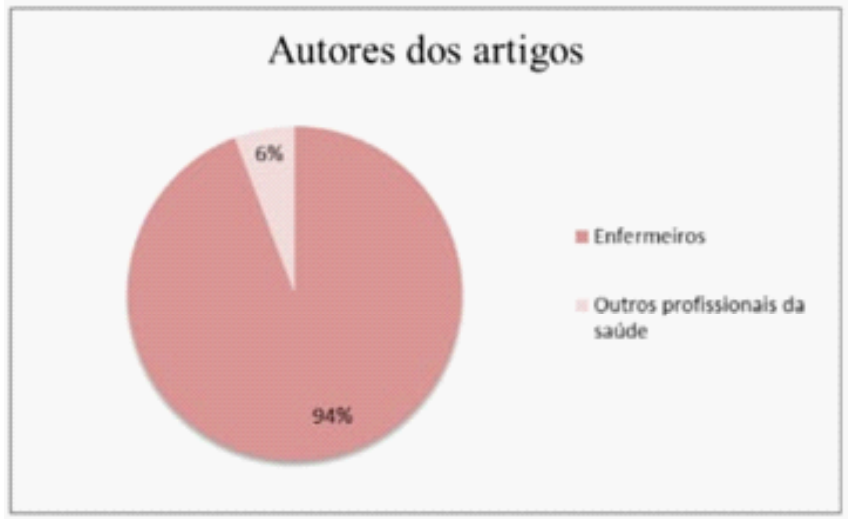

Figura 1. Sobre os autores dos artigos.

Fonte: Autor, 2021.

De acordo com os dados apresentados, a maioria dos artigos utilizados foram produzidos por profissionais da aréa da enfermagem.

Percebeu-se a necessidade de estender pesquisas com o tema em questão, para o aprimoramento do conhecimento, que até então é pouco debatido pelos profissionais da aréa da saúde. Pois somente através do conhecimento podemos buscar soluções por meio de ações conjuntas com o Estado e a sociedade. ${ }^{16}$

Por estarem presentes em diversos níveis da atenção à saúde, os profissionais de enfermagem são essenciais para realizar a identificação e a prevenção de violações contra a integridade da pessoa idoso. ${ }^{17}$ Estes devem estar aptos para identificar qualquer sinal que indique maus-tratos, realizar corretamenta a conduta de intervenção e o acolhimento à vítima. Entre os diversos fatores intrínsecos que dificultam o enfermeiro a realizar a notificação dos casos, é o idoso não querer denunciar $o$ agressor por questões de afinidade, medo e vergonha. ${ }^{2}$ Embora a maioria dos casos sejam de difícil diagnóstico devido a violência ser omitido pela própria vítima, os estudos analisados demonstram que os profissionais mais qualificados e que recebem incentivos, são os que estão mais preparados e realizam a identificação precoce dos casos de agressões. ${ }^{18}$

O profissional de saúde, em específico a equipe da visita domiciliar, tem um papel importante ao terem contato direto com o paciente, sendo a modalidade mais propícia para a evidenciação dos casos de violência 
contra os idosos e suas vulnerabilidades. A partir das visitas é possível conhecer a realidade vivenciada pelo idoso em seu domícilio. Nos casos identificados, as ações implementadas pelos enfermeiros consistem em: orientar os idosos, familiares e cuidadores por meio de ações educativas, acionar a delegacia e encaminhar para os serviços sociais disponíveis. ${ }^{18}$

As percepções dos participantes são claras de que a violência vivida pelos idosos hoje se deve à história familiar, conflitos e desentendimentos vividos em famílias acostumadas a relacionamentos não saudáveis e ciclos contínuos de violência. A descoberta da violência não faz com que os profissionais se vejam como agentes de mudança da realidade, pois para eles a violência se explica pelo individualismo e pelo modo de vida capitalista. Os idosos são vistos como um elemento sem inutilidade pela sociedade. Além disso, a ampliação e criação de novos programas educacionais de incentivo à valorização dos idosos nas escolas e demais instituições de controle social é fundamental para reconstruir o sentido da velhice para as próximas gerações e proporcionar uma realidade futura menos amarga do que a vivenciada atualmente por muitos idosos. ${ }^{19}$

Nesse sentido, o enfermeiro por estar em convivência contínua com o público da terceira idade, deve aproveitar as oportunidades para investigar situações de agressões, desenvolver estratégias de identificação e enfrentamento para o problema através dos instrumentos validados e da escuta atenta durante a consulta de enfermagem, além de promover a prevenção como divulgadores das informações relativas à violência. ${ }^{19}$

\section{Políticas públicas e os direitos do idoso}

Com o intuito de defender a pessoa idosa, faixa etária da população com maior crescimento nos últimos anos, em 1994 foi promulgada a Lei ${ }^{\circ}$. 8.842/94, que criou a Política Nacional do Idoso, que foi um amadurecimento nas leis relacionadas ao idoso. As diretrizes da Política Nacional do Idoso são as seguintes:

"Lei 8.842/94. Art. $3^{\circ}$. Priorização do atendimento familiar ao idoso e não o seu recolhimento a asilos, exceto quando o idoso é sozinho; busca de opções de integração entre os idosos e as demais gerações; participação do idoso no planejamento, desenvolvimento, implementação e avaliação de políticas, projetos, planos eprogramas deseuinteresse; descentralização política administrativa; reciclagem e capacitação de novos profissionais nas áreas de geriatria, gerontologia e prestação de serviços; implementação de sistemas de informações que divulguem de forma educativa os aspectos biopsicossociais do envelhecimento; priorização de serviços públicos e privados prestadores de serviços; apoio a estudos e pesquisas sobre questões relativas ao envelhecimento." 20
Em 2002, o Conselho Nacional da Terceira Idade foi instituído por meio do Decreto $\mathrm{n}^{\circ} 16$. O documento $\mathrm{n}^{\circ} 4.227$ é uma importante ferramenta para a proteção do idoso, além de fornecer orientações gerais para a proteção do idoso no Brasil por meio do "Manual do Idoso". Quase dez anos após a promulgação da Política Nacional de Terceira Idade, o artigo 10.741 , de $1^{\circ}$ de outubro de 2003, define a terceira idade como "pessoa de 60 anos, resguardando-lhes direitos semelhantes aos direitos conferidos à criança e ao jovem, que incluem o direito à proteção adequada." Assim, foi aprovado em 2003 o Estatuto do Idoso, visando: regular, garantir os direitos e estipular responsabilidades. ${ }^{21}$

A promulgação legislativa do Estatuto do Idoso representa um marco na concretização dos direitos básicos das pessoas idosas. O Estatuto é mais abrangente do que a Política Nacional do Idoso e impõe penalidades severas a quem desrespeitar ou abandonar o idoso.

Em fevereiro de 2006, o Regulamento n ${ }^{\circ}$ 399/GM emitiu o documento de orientação "Pacto de Saúde", que envolvia a "Pacto de vida". Neste documento, a saúde do idoso aparece como uma das seis prioridades pactuadas pelo governo em três áreas, e uma série de ações foram realizadas para finalmente implementar as diretrizes da Política Nacional de Saúde da Pessoa Idosa. $^{21}$

\section{Conclusão}

O estudo analisou a assistência do enfermeiro (a) na identificação e prevenção da violência contra o idoso. Percebeu-se a necessidade de se treinar os profissionais da área de saúde para que estejam preparados quando a violência for detectada.

Ressalta-se, ainda, no estudo presente as leis e as políticas públicas que resguardam o idoso.AConstituição e o Estatuto do Idoso representam um importante passo na promoção dos direitos da pessoa idosa, no qual prever penas para os casos de descumprimento às normas estatutárias e obrigatoriedade da denúncia de maus-tratos por profissionais de saúde.

Portanto, a violência contra os idosos constitui uma violação dos direitos humanos, e exige que o poder público e a sociedade tomem ações estratégicas de prevenção e resposta para resgatar e garantir a dignidade dessas pessoas. Buscando construir uma sociedade mais justa e humana para todos.

Considera-se que os objetivos desta pesquisa foram alcançados, contribuindo assim para a prática da enfermagem.

\section{Referências}

1. Perissé C, Marli M. Revista Retratos a revista do ibge, 2019 fev.;3(16):18-20. 
2. Azevedo CO, Silva TASM. Cuidados de Enfermagem para detecção de violência contra idosos. Revista Pró-UniverSUS, 2019 jan./jun.;10(1):5559.

3. Oliveira MLC, Gomes ACG, Amaral COM, Santos LB. Características dos idosos vítimas de violência doméstica no Distrito Federal. Revista Brasileira Geriatria e Gerontologia, 2012 jul./set.;15(3):55-66.

4. Alarcon MFS, Damaceno DG, Cardoso BC, Sponchiado VBY, Braccialli LAD, Marin MJS. Percepção do idoso acerca da violência vivida. Revista Baiana de Enfermagem, 2020; 34:e34825

5. Moraes CLD, Marques ES, Ribeiro AP, Souza ERD. Violência contra idosos durante a pandemia de Covid-19 no Brasil: contribuições para seu enfrentamento. Ciência \& Saúde Coletiva, 2020; 25:4177-4184.

6. Rocha EM, Vilela ABA, Oliveira DC, Silva DM, Alves MR, Meira SS. Estrutura representacional de profissionais da estratégia da família sobrea a violência intrafamiliar contra idosos. Revista de enfermagem UERJ, 2015 mar./abr.;23(2):178-84

7. Rocha EN, Vilela ABA, Silva DM. Enfretamento da violência intrafamiliar contra pessoas idosas pelos profissionais de saúde. Revista Kairós; 2015 nov.;18(4):29-46.

8. Oliveira SC; Leite AC; Monteiro LC; Pavarini SCL. Violência em idosos após a aprovação do Estatuto do Idoso: revisão integrativa. Revista Eletrônica de enfermagem, 2012 jun.;14(4):974-982.

9. Moura LBA, Noronha VMADS, Vieira ABD, Faustino AM. Percepções de qualidade de vida e as experiências de violências em idosos. Revista de enfermagem UFPE on line, 2018 ago.; 12(8):2146-2153.

10. Oliveira AAVD, Trigueiro DRSG, Fernandes MDGM, Silva AO. Maus-tratos a idosos: revisão integrativa da literatura. Revista Brasileira de Enfermagem, 2013 jan./fev.; 66(1):128-133.

11. Polaro SHI, Gonçalves LHT, Alvarez AM. Enfermeiras desafiando a violência no âmbito de atuação da estratégia de saúde da família. Revista Texto Contexto Enfermagem, 2013 out./dez.; 22(4):935-42.

12. Lopes EDS, Ferreira AG, Pires CG, Moraes MCS, D’elboux MJ. Maus-tratos a idosos no Brasil: uma revisão integrativa. Revista Brasileira de Geriatria e Gerontologia, 2018;21(5):628-638.

13. Rodrigues TP, Moreira MASP, Silva AO, Smith ADAF, Almeida JLTD, Lopes MJ. Sentidos associados à violência para idosos e profissionais. Escola Anna Nery Revista Brasileira de Enfermagem, 2010, 14(4):772-778.

14. Musse JDO, Rios MHE. Atuação do enfermeiro perante a violência doméstica sofrida pelo idoso. Revista Estudos Interdisciplinares sobre o envelhecimento, 2015 dez.;20(2): 365-379

15. Antequera IG, Lopes MCBT, Batista REA, Campanharo CRV, Costa PCPD, Okuno MFP. Rastreamento de violência contra pessoas idosas: associação com estresse percebido e sintomas depressivos em idosos hospitalizados. Escola Anna Nery Rev. Enfermagem, 2020 nov.;25(2):e20200167.

16. Oliveira KSM, Carvalho FPB, Oliveira LC, Simpson CA, Silva FTL,Martins AGC. Violência contra idosos: concepções dos profissionais de enfermagem a cerca da detecção e prevenção. Revista Gaúcha de Enfermagem, 2018 jul.;39(2):e57462

17. Almeida CAPL, Neto MCS, Carvalho FMFD, Lago EC. Aspectos relacionados à violência contra o idoso: concepcão do enfermeiro da Estratégia Saúde da Família. Revista de Pesquisa: Cuidado é Fundamental, 2016;11(2):404-410

18. Castro VC, Rissardo LK, Carreira L. Violência contra os idosos brasileiros: uma análise das internações hospitalares. Revista Brasileira de Enfermagem, 2018;71(2): 777-785.

19. Winck DR, Alvarez AM. Percepções de enfermeiros da estratégia saúde da família acerca das causas da violência contra a pessoa idosa. Revista APS, 2018 jan./mar.; 21(1):93-103.

20. Brasil. Lei $n^{\circ}$. 8.842/94. Política Nacional do Idoso. 04 de Janeiro de 1994. Disponível em: http://www.planalto.gov.br/lei

21. Moreira WC, Damasceno CKCS, Vieira SKSF. Análise sobre as políticas públicas de enfrentamento a violência contra o idoso. Revista de 